
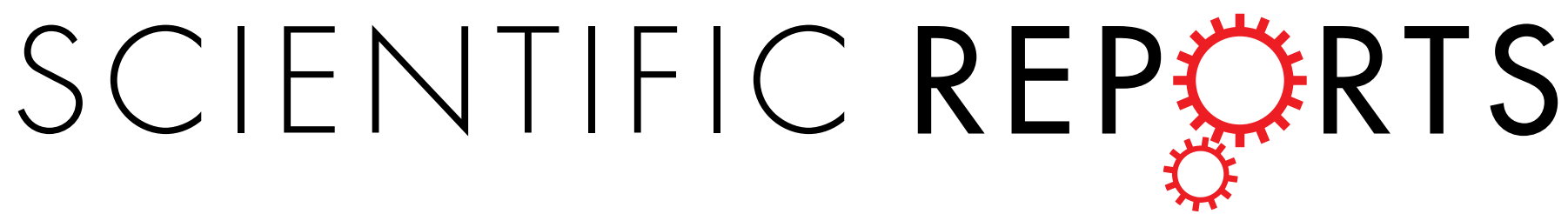

\title{
OPEN Functional validation of GPIHBP1 and identification of a functional mutation in GPIHBP1 for milk fat \\ traits in dairy cattle
}

Received: 16 August 2016

Accepted: 12 July 2017

Published online: 17 August 2017
JieYang, Xuan Liu, Dan Wang, Chao Ning, Haifei Wang, Oin Zhang \& Li Jiang

In a previous genome-wide association study (GWAS) on milk production traits in a Chinese Holstein population, we revealed that GPIHBP1 is a novel promising candidate gene for milk fat content traits. In this study, we performed over-expression and RNAi experiments on GPIHBP1 in bovine primary mammary epithelial cells. The results showed that the expression of several important milk fatrelated genes ( $L P L, C D 36, V L D L R, A C A C A$ and $F A S N)$ increased or decreased when the expression of GPIHBP1 was up- or down-regulated. To identify the potential functional SNP involved, we explored the genetic variants of GPIHBP1 and found that a G/A mutation (chr14:2553998) in the promoter region of GPIHBP1 significantly reduced promoter activity and had an effect on transcription factor binding sites. This finding was consistent with the lower expression of GPIHBP1 observed in the mammary gland tissue of cows harboring the homozygous AA mutation compared with wild-type homozygous GG or heterozygous AG. Furthermore, association analysis showed that cows with the AA genotype outperformed those with the $G G$ and $A G$ genotypes in terms of the milk fat percentage. Our study demonstrates that GPIHBP1 could be a strong candidate gene for milk fat content traits and, in particular, the $\mathrm{G}$ to $A$ mutation at chr14:2553998 within GPIHBP1 could be a functional mutation related to its effects.

GPIHBP1 (glycosylphosphatidylinositol-anchored high-density lipoprotein binding protein 1) is a member of the lymphocyte antigen 6 (Ly6) family ${ }^{1}$. Recent studies showed that GPIHBP1 plays a critical role in the transportation and localization of lipoprotein lipase (LPL) and serves as a platform for lipolysis in endothelial cells ${ }^{2-7}$. GPIHBP1 also interacts with LPL and represents an important binding site for LPL in vivo ${ }^{8}$. LPL, which is synthesized in the mammary gland and mediates lipolytic processing within the mammary gland, is important for providing lipid nutrients to produce milk fat ${ }^{9,10}$. Thus, GPIHBP1 is also involved in the process of milk fat synthesis. The importance of GPIHBP1 in triglyceride metabolism was demonstrated by Beigneux et al. ${ }^{11}$ in mice, who showed that GPIHBP1-knockout (GPIHBP1-/-) mice displayed severe hypertriglyceridemia, even on a low-fat diet, exhibiting a plasma triglyceride level of $1000-6000 \mathrm{mg} / \mathrm{dl}$ at $7-10$ weeks of age.

In a previous genome-wide association study (GWAS) ${ }^{12}$ and the subsequent analysis of the novel variants revealed by targeted sequencing of GWAS loci ${ }^{13}$, we found that an SNP in the promoter region of GPIHBP1 showed a very strong association with the milk fat percentage, with a $P$ value of $5.0 \mathrm{E}-18$. We subsequently detected the mRNA expression levels of GPIHBP1 in eight different tissues of lactating cows. The results revealed that the mRNA expression level of GPIHBP1 in the mammary gland was much higher than that in the seven other tissues $^{13}$. These results, together with the known biological function of GPIHBP1 revealed in humans and mice, suggest that GPIHBP1 is a promising candidate gene for milk fat traits in dairy cattle.

The objective of this study was to validate the effect of GPIHBP1 on milk fat traits in dairy cattle and to identify the potential functional mutation involved. We investigated the genetic variants of GPIHBP1 via sequencing, identified four SNPs in the promoter region, and then performed an association analysis of these SNPs and the milk fat percentage in a Chinese Holstein cattle population that was different from the populations used in our previous studies ${ }^{12,13}$. We subsequently investigated the expressional relationship between GPIHBP1 and some

Key Laboratory of Animal Genetics, Breeding and Reproduction, Ministry of Agriculture \& National Engineering Laboratory for Animal Breeding, College of Animal Science and Technology, China Agricultural University, 100193, Beijing, P.R. China. Correspondence and requests for materials should be addressed to L.J. (email: lijiang@cau.edu.cn) 
A

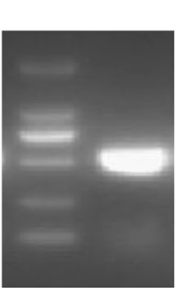

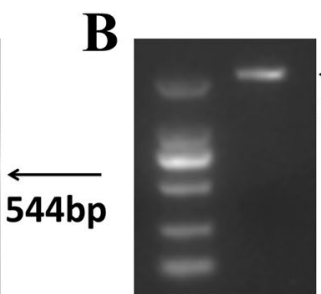
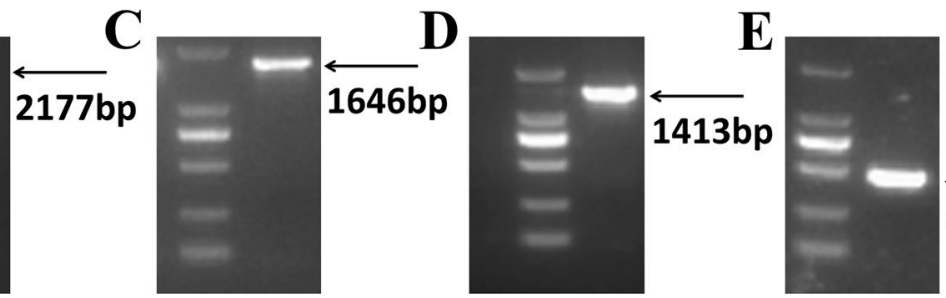

$482 \mathrm{bp}$

Figure 1. PCR amplification of the open reading frame (ORF) and different promoter segments of GPIHBP1. The DNA markers were DL2000:2000bp, 1000 bp, 750 bp, 500 bp, 250 bp, 100 bp. (A) Band of the ORF $($ size $=544 \mathrm{bp})$. (B) Band of the promoter fragment ( $\operatorname{cize}=2177 \mathrm{bp}$ ) that contains all four SNPs. (C) Band of the promoter fragment (size $=1646 \mathrm{bp}$ ) that contains the first three SNPs. (D) Band of the promoter fragment $($ size $=1413 \mathrm{bp})$ that contains the first two SNPs. $(\mathrm{E})$ Band of the promoter fragment $($ size $=482 \mathrm{bp})$ that contains only the first SNP.

known milk fat-related genes by over-expressing and down-regulating GPIHBP1 in bovine primary mammary epithelial cells. To identify the potential functional SNP involved, we tested the effects of the four SNPs on promoter activity via a dual-luciferase reporter system and the effects on transcription factor binding via gel retardation assays. Our results provide valuable information for elucidating the genetic basis of milk fat traits.

\section{Results}

Exploring genetic variants of GPIHBP1. We explored the genetic variants of GPIHBP1 via sequencing and identified four SNPs (G/A at chr14:2553998, C/A at chr14:2553653, G/A at chr14:2553525, and A/G at chr14:2552574) in the promoter region, one synonymous mutation (C/T at chr14:2550469) in the first exon, one SNP (G/T at chr14:2550326) in the $3^{\prime}$ UTR region, and one SNP (G/C at chr14:2551922) in the third intron. Because knowledge regarding the functions of mutations in introns and synonymous mutations in exons is still limited, and significant SNPs for milk fat traits were found only in the promoter region in our previous work ${ }^{12,13}$, we focused our attention on the mutations in the promoter region. We genotyped the four SNPs in 158 randomly selected individuals via PCR and performed haplotype analysis using PHASE v2.1.1 ${ }^{14,15}$. The four SNPs were completely linked, and only two haplotypes, GCGA and AAAG, were detected. Among the 158 individuals, 31 were homozygous for the GCGA haplotype, 47 were homozygous for the AAAG haplotype, and 80 were heterozygous.

Association analysis. An association analysis was performed to test the effects of these SNPs on milk fat and the relationship between their effect and the effect of DGAT1 K232A. Because these SNPs were completely linked with each other, we analyzed only one of them, chr14:2553998. A total of 6619 cows from a different population than those employed in our previous studies ${ }^{12,13}$ were used in the analysis. The results showed that when only chr14:2553998 was fit in the model, the $P$ value was 1.13E-35. The cows with genotype AA, homozygous for the mutant allele A, exhibited a significantly higher milk fat percentage than cows with genotypes GG and GA (least squares means $=1.24,-10.57$ and -4.80 , respectively). When fitting only K232A in the model, the $P$ value was 8.9E-127, and the least squares means of the three genotypes, AA, GG, and AG, were 17.48, -10.56 , and 4.13, respectively. When fitting both SNPs in the model, the $P$ values for chr14:2553998 and K232A were 0.0029 and 7.4E-95, respectively. The least squares means for chr14:2553998 were -2.70 (AA), -6.73 (GG), and -4.40 (AG), and those for K232A were 16.75 (AA), -10.36 (GG), and 3.85 (AG). The interaction between the two loci was not significant $(P>0.4)$.

Haplotype analysis between K232A and chr14:2553998. The haplotype analysis between K232A and chr14:2553998 revealed an linkage disequilibrium (LD) level of $r^{2}=0.137$ between these SNPs, and the frequencies of the four haplotypes, G(K232A)-G(2553998), A((K232A)-A(2553998), G(K232A)-A(2553998), and $\mathrm{A}(\mathrm{K} 232 \mathrm{~A})-\mathrm{G}(2553998)$, were $40.6 \%, 19.7 \%, 38.2 \%$, and $1.4 \%$, respectively.

Over-expression of GPIHBP1 in BMECs. We constructed a recombinant pcDNA3.1(+)-GPIHBP1 eukaryotic expression vector. The PCR products were loaded into $2 \%$ agarose gels, and the band size of the open reading frame (ORF) of GPIHBP1 was $544 \mathrm{bp}$ (Fig. 1A). After the ORF was inserted into the pcDNA 3.1(+) plasmid, the pcDNA 3.1(+)-GPIHBP1 plasmid sequence was confirmed through DNA sequencing. Then, we transiently transfected the vector into bovine primary mammary epithelial cells (BMECs). After transfection, the mRNA expression level of GPIHBP1 was analyzed via quantitative RT-PCR. The results showed that the expression of GPIHBP1 was significantly increased $(P<0.001)$ compared with that in control cells (transfected with the empty vector pcDNA 3.1(+)) (Fig. 2). We then detected the mRNA expression levels of five milk fat-related genes (LPL, $V L D L R, C D 36, A C A C A$, and FASN) and found that their mRNA expression levels were all increased (Fig. 2), with the changes in VLDLR, ACACA, and FASN being significant $(P<0.05)$.

siRNA-mediated silencing of GPIHBP1 in BMECs. We transfected BMECs with Stealth ${ }^{\mathrm{TM}}$ RNAi siRNA targeting the bovine GPIHBP1 gene open reading frame. After 48 hours, the GPIHBP1 mRNA level in BMECs was significantly decreased $(P<0.05)$ compared with that in control cells (transfected with Stealth ${ }^{\mathrm{TM}}$ RNAi siRNA 

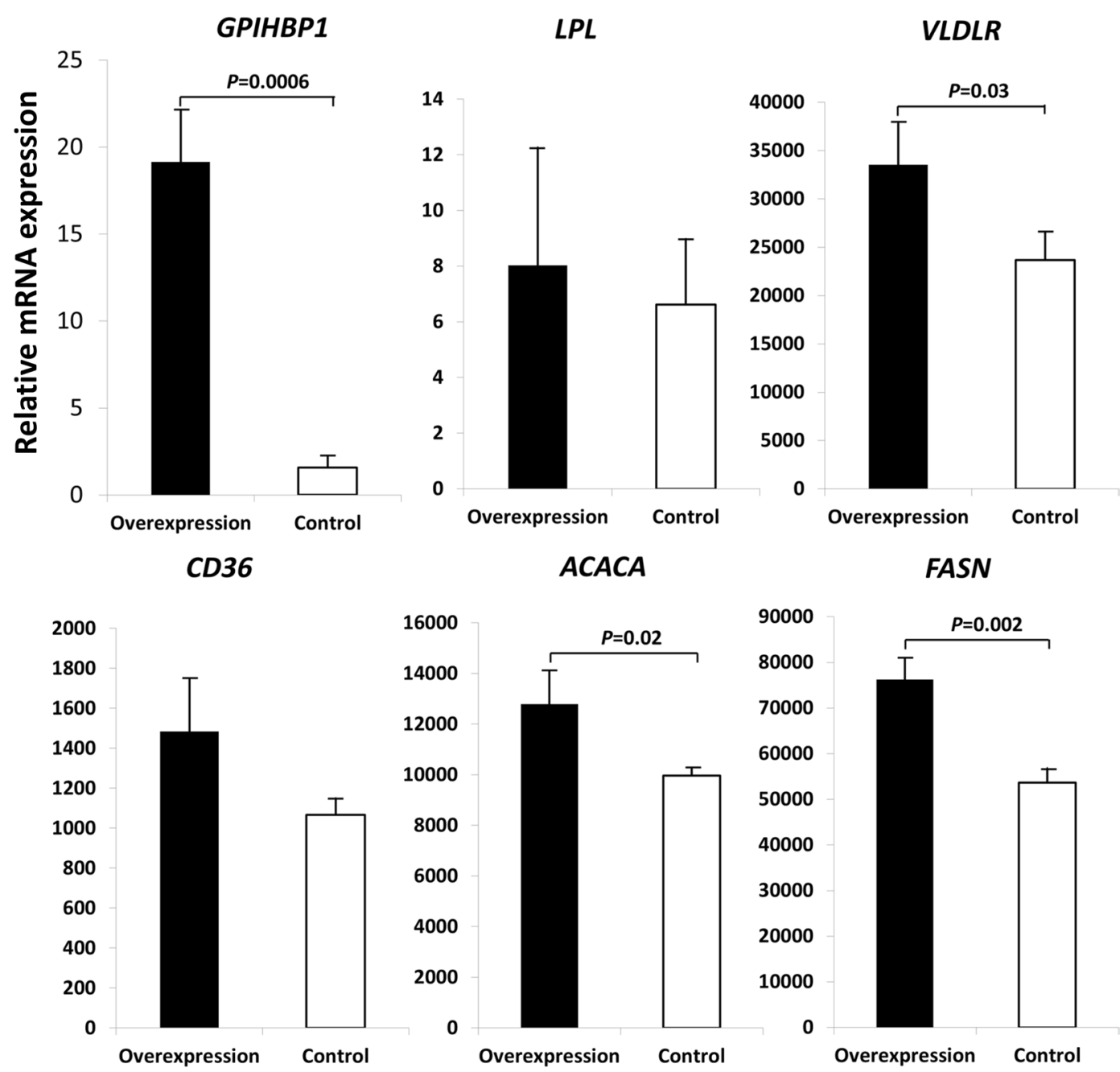

Figure 2. Expression of GPIHBP1 and five milk fat-related genes in bovine primary mammary epithelial cells that were transfected with the pcDNA3.1(+)-GPIHBP1 eukaryotic expression vector (over-expression) and with an empty vector pcDNA 3.1(+) (control). The vertical axes represent the expression of these genes relative to the expression of the housekeeping gene GAPDH.

Negative Control) (Fig. 3). The mRNA expression levels of the five fat-related genes ( $L P L, V L D L R, C D 36, A C A C A$ and FASN) were all reduced (Fig. 3), with the changes in $L P L, A C A C A$, and FASN being significant $(P<0.05)$.

Effects of SNPs on the promoter activity of GPIHBP1. To detect the effects of the four SNPs in the promoter region of GPIHBP1 on promoter activity, we constructed eight recombinant vectors carrying the GPIHBP1 promoter corresponding to the four SNPs and the wild-type and mutant haplotypes and then measured the promoter activity of different segments using a dual-luciferase reporter system. The band sizes for the GPIHBP1 promoter fragments corresponding to the four SNPs were $482 \mathrm{bp}(\mathrm{P} 1), 1413 \mathrm{bp}$ (P2), 1646bp (P3), and $2177 \mathrm{bp}$ (P4), respectively (Fig. 1B-E). All the recombinant promoter plasmids exhibited a stronger luciferase response than the negative control (pGL4.14 vector) (Fig. 4), showing the normal promoter activity of the inserted fragments. However, for P1, P2 and P3, the mutant type displayed a stronger luciferase response than the wild type $(P<0.01)$, while for $\mathrm{P} 4$, the wild type showed greater luciferase activity than the mutant type $(P<0.01)$. These results suggested that the fourth SNP, G/A (chr14:2553998), might be the functional mutation that significantly reduces the promoter activity of GPIHBP1, leading to decreased mRNA expression of GPIHBP1.

To confirm the causality of the G/A SNP at chr14:2553998 for the decreased mRNA expression of GPIHBP1, it is necessary to show its effect on the promoter activity of GPIHBP1 on the identical haplotypic background for the other three SNPs. Since the four SNPs were completely linked and there were only two haplotypes (GCGA and AAAG) in our experimental population, we reconstituted two haplotypes (ACGA and GAAG) using site directed mutagenesis in vitro. Then, we compared the promoter activities of GPIHBP1 of four different promoter vectors with respect to the four haplotypes (GCGA, ACGA, GAAG, and AAAG). Figure 5 clearly shows that for both haplotypic background AAG and CGA, the mutation G to A at chr14:2553998 significantly reduced $(P<0.01)$ the promoter activity of GPIHBP1. These results demonstrated that $\mathrm{G}$ to A at chr14:2553998 is the causal mutation leading to the decreased promoter activity of GPIHBP1, while the mutations at the other three SNPs increased the activity, which is consistent with the results showing in Fig. 4. 
GPIHBP1

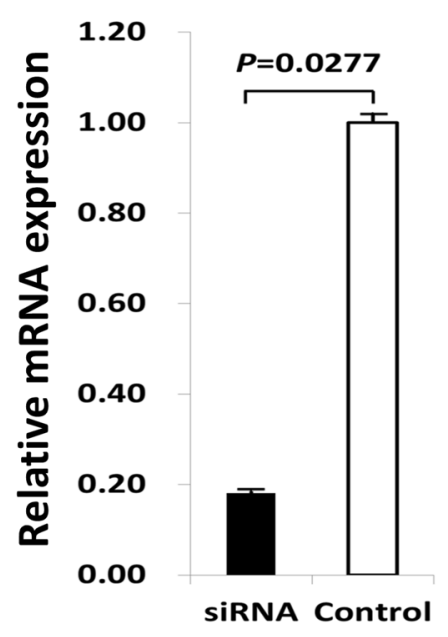

CD36

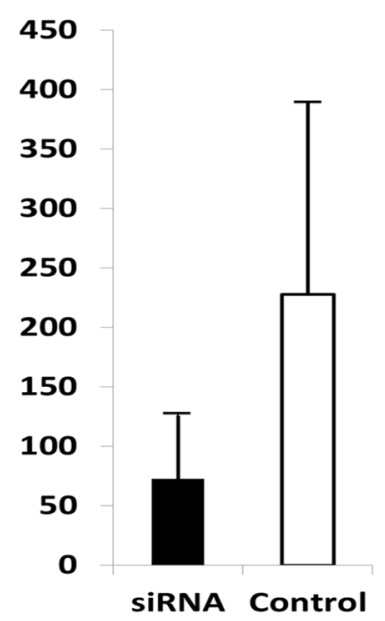

$\boldsymbol{L P L}$

500

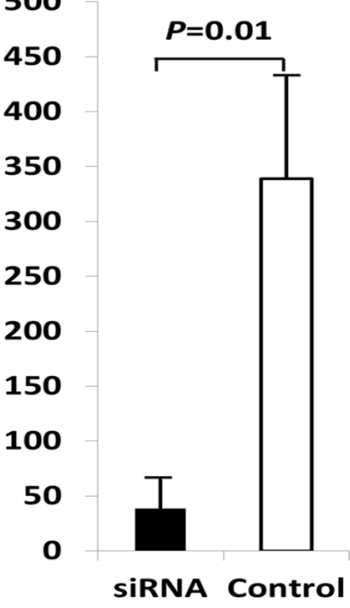

ACACA

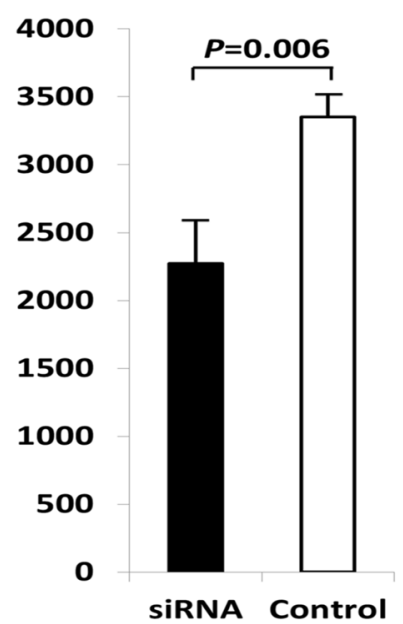

VLDLR

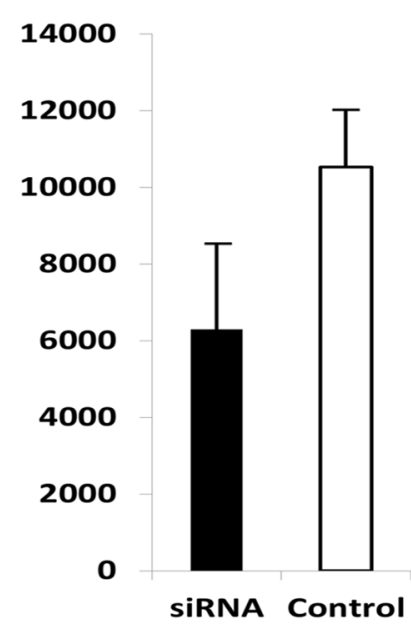

FASN

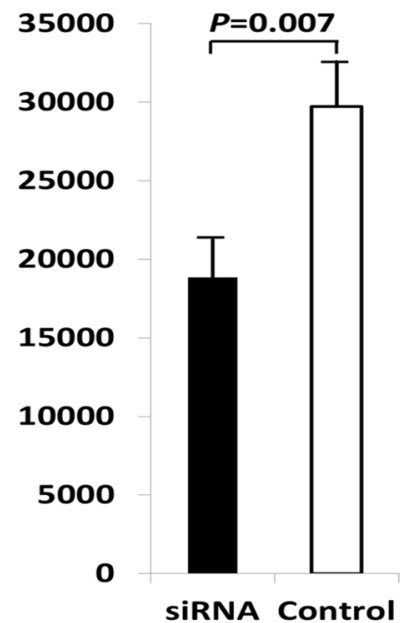

Figure 3. Expression of GPIHBP1 and five milk fat-related genes in bovine primary mammary epithelial cells that were transfected with Stealth ${ }^{\mathrm{TM}}$ RNAi siRNA targeting the bovine GPIHBP1 gene open reading frame (siRNA) and with the Stealth ${ }^{\mathrm{TM}}$ RNAi siRNA Negative Control (control). The vertical axes represent the expression of these genes relative to the expression of the housekeeping gene GAPDH.

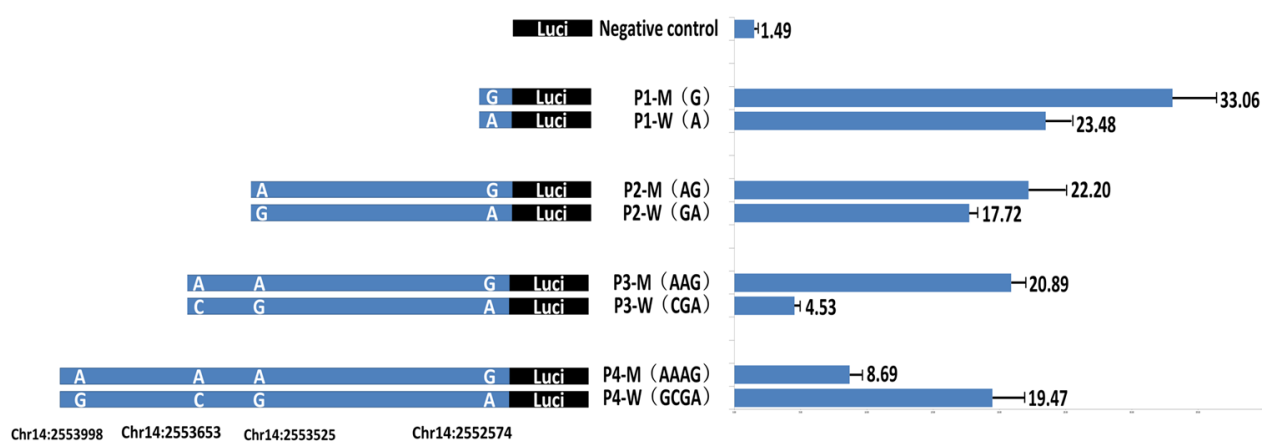

Figure 4. Promoter activity analysis of the bovine GPIHBP1 gene. There are four completely linked SNPs in the promoter region of GPIHBP1. As shown, fragments P1, P2, P3, and P4 contain one, two, three, and four SNPs sites, respectively. We constructed eight types of recombinant promoter vectors with respect to the four fragments and the mutant and wild-type haplotypes. Promoter activities were detected using a dual-luciferase reporter system. The data are expressed as the means and standard errors of three replicates. 


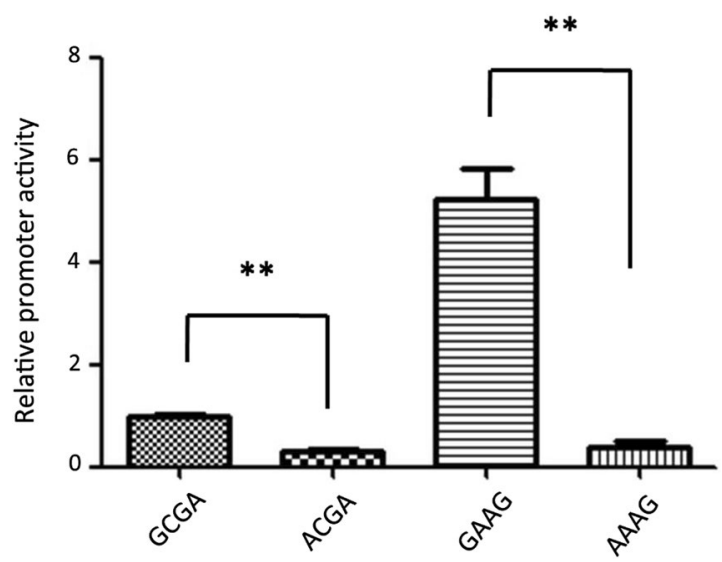

Figure 5. Relative promoter activities of GPIHBP1 of four different promoter vectors with respect to the four haplotypes (GCGA, ACGA, GAAG, and AAAG). Promoter activities were detected using a dual-luciferase reporter system. The data are expressed as the means and standard errors of three replicates $* * P<0.01$.

\section{wild probe mutation probe}

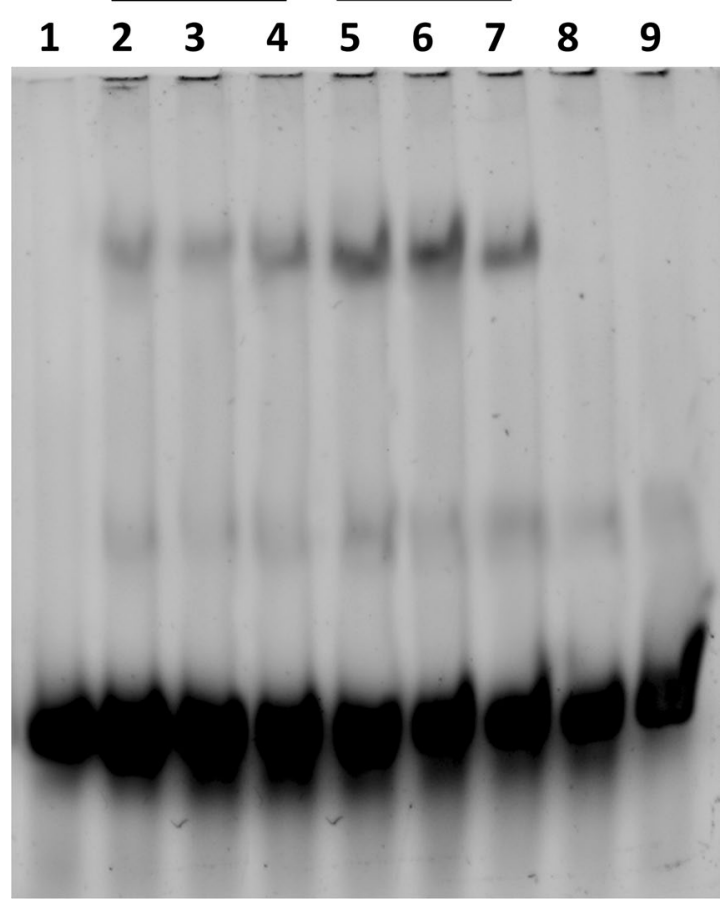

Figure 6. Characterization of the protein binding site for the SNP site, G/A (chr14:2553998), revealed in EMSA experiments. (A) Lane 1= blank; Lanes 2, 3, 4= nuclear extract + biotin-labeled Probe-wild type; Lanes 5, 6, $7=$ nuclear extract + biotin-labeled Probe-mutant type; Lane $8=$ nuclear extract +100 times of cold Probe-wild type; Lane $9=$ nuclear extract +100 times of cold Probe-mutant type. The probes with the mutant allele showed higher nuclear protein affinity than the probe with the wild-type allele.

In addition, we predicted the transcription factor binding sites of the G/A SNP at chr14:2553998 using Genomatix (http://www.genomatix.de/). The results showed that this mutation could influence the binding sites for transcription factors (Table S1). To confirm this prediction, we performed an electrophoretic mobility shift assay. As shown in Fig. 6, without pre-incubation of the nuclear extract with cold probes, protein and labeled probe complexes formed for both the mutant probe and wild-type probe, which retarded the movement of the probe in the gel. However, the nuclear protein showed a higher affinity for the probe with the mutant allele (A) than the probe with the wild-type allele $(G)$, indicating that the mutation had an effect on the binding sites of transcription factors and could indeed regulate the promoter activity of GPIHBP1. When the nuclear extracts were incubated in the presence of a 100-fold concentration of the cold probes, protein binding to the labeled probe was abolished, as shown by the lack of visible protein-probe complexes in the gel shift assay. 


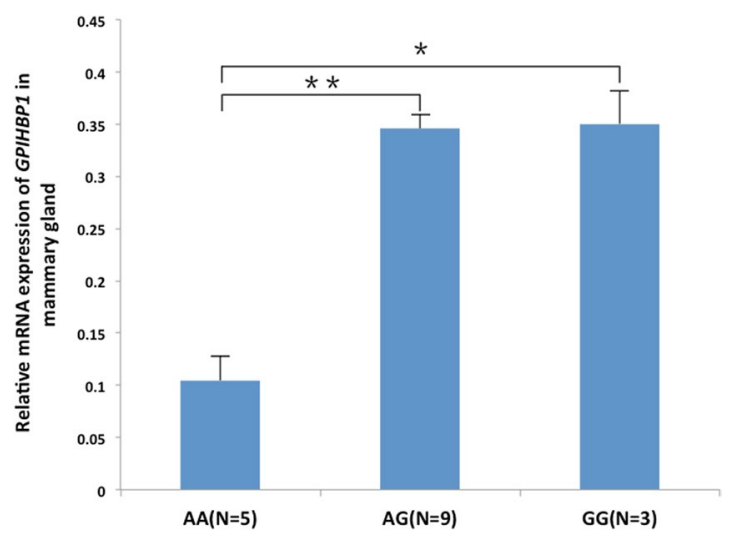

Figure 7. Expression of GPIHBP1 in the mammary gland of cows with genotypes AA, GG, and GA.

\section{Discussion}

In a previous study ${ }^{13}$, we revealed a strong association $(P=5.0 \mathrm{E}-18)$ between the SNP chr14:2553525 (SNP P89 in that paper) in the promoter region of GPIHBP 1 and the milk fat percentage. However, this SNP is only $751 \mathrm{~kb}$ away from the K232A mutation in DGAT1, which is well known to have major effects on milk fat traits, with a LD level of $r^{2}=0.148$. When K232A was fixed in the analysis model, this association became non-significant $(P=0.0566)$, suggesting that this association might be due to LD between chr14:2553525 and K232A. To clarify this issue, we analyzed the association of chr14:2553998 and K232A with the fat percentage in another population with 6619 cows. Because chr14:2553998 is completely linked with chr14:2553525 (as shown in this study), these SNPs should have the same effect on the milk fat percentage. When chr14:2553998 and K232A were separately fitted in the model, both of the SNPs showed a very strong association with the milk fat percentage. When both of them were fit in the model simultaneously, chr14:2553998 still showed a highly significant association, although the $P$ value increased markedly, and the interaction between the SNPs was not significant. These results suggest that the SNP chr14:2553998 contributes to the phenotypic variation of the milk fat percentage, although its effect is largely masked by K232A. Haplotype analysis between the two loci revealed that the majority (over 60\%) of haplotypes were formed by the favorable (or unfavorable) alleles of the two loci, suggesting that the legitimate effects of GPIHBP1 and DGAT1 could be driven by the same haplotype. Moreover, from the point of view of the known biological functions of the two genes, DGAT1 is a key enzyme in triacylglycerol synthesis, catalyzing the final and only committed step in triglyceride synthesis ${ }^{16}$, while GPIHBP1 is a key element in the binding and transport of lipoprotein lipase (LPL) $)^{2-7}$, which is essential for providing lipid nutrients to produce milk fat ${ }^{9,10}$. Thus, both DGAT1 and GPIHBP1 play important roles in triglyceride synthesis, either separately or interactively.

To clarify the effect of GPIHBP1 on milk fat traits, we investigated the effects of over-expressing and silencing GPIHBP1 on the expression of several milk fat-related genes ( $L P L, V L D L R, C D 36, A C A C A$, and FASN). We demonstrated that following the over-expression or silencing of GPIHBP1, the mRNA expression of all of these genes was either increased or decreased. The GPIHBP1 protein localizes to the membrane of epithelial cells, where it can bind with LPL and chylomicrons, providing a platform for the lipolysis of triglyceride in chylomicrons by $\mathrm{LPL}^{17-20}$. The released non-esterified fatty acids (NEFAs) will be transported into cells by CD36 and VLDLR and used as substrates of ACACA and FASN, both of which are important enzymes in fatty acid synthesis ${ }^{17,21-23}$. When the expression of GPIHBP1 is decreased, the binding of LPL with GPIHBP1 is also decreased, such that the process of triglyceride lipolysis in mammary epithelial cells is inhibited, leading to increased accumulation of triglycerides and, thus, a higher milk fat content. Additionally, the reduced release of NEFAs will lead to decreased expression of CD36 and VLDLR. In addition, the expression of ACACA and FASN will also decrease due to the reduction of NEFAs. Our findings following siRNA knockdown GPIHBP1 as well as over-expression of GPIHBP1 were fully consistent with these analyses.

Four SNPs were identified in the promoter region of GPIHBP1, which were completely linked with each other. Promoter activity analysis using recombinant promoter vectors revealed that all four SNPs appeared to have effects on promoter activity (Fig. 4). Interestingly, however, the fourth SNP (chr14:2553998), showed an opposite effect compared with the other three SNPs; i.e., the mutant allele of this SNP significantly reduced promoter activity, while the mutant alleles of the other three SNPs increased promoter activity. Through transcription factor binding site prediction and electrophoretic mobility shift assays, we found that the mutation at chr14:2553998 affected binding sites for transcription factors and might regulate the transcript expression level of the GPIHBP1 gene. To test this possibility, we performed qPCR analysis to detect the expression levels of different genotypes of the SNP chr14:2553998 in the mammary glands of 17 cows. The results showed that the expression of the homozygous mutant (AA) was significantly lower than that of the other two genotypes (Fig. 7), which is consistent with the observation that the mutant allele of this SNP reduced promoter activity. To verify the causality of this mutation for the reduction of the GPIHBP1 expression and whether its effect is confounded by the genotypes of the other three SNPs, we compared the promoter activities of GPIHBP1 with varied alleles (G and A) at this SNP on identical haplotypic background (either AAG or CGA) at the other three SNPs. It turned out that with both haplotypic backgrounds the mutant allele A significantly reduced the promoter activity of GPIHBP1 compared to allele G. Thus, we believe that the chr14:2553998 SNP should be the causal mutation responsible for the reduction of GPIHBP1 expression. Moreover, the association analysis between the genotypes of this SNP and the 
milk fat percentage also showed that cows with genotype AA exhibited a higher milk fat percentage than cows with genotypes GG and GA $(P<0.001)$. The effect of this SNP on the milk fat percentage could be caused by the decreased mRNA expression of GPIHBP1.

In conclusion, we showed that among the four completely linked SNPs identified in the promoter region of GPIHBP1, the $\mathrm{G}$ to A mutation at chr14:2553998 could cause the reduction the promoter activity of GPIHBP1, leading to decreased expression of GPIHBP1. We also showed that the decreased expression of GPIHBP1 could lead to decreased expression of $L P L$, the gene encoding the LPL enzyme responsible for triglyceride lipolysis. Thus, the higher milk fat percentage associated with the mutant A allele of this SNP could be explained by reduced triglyceride lipolysis and increased accumulation of triglyceride. Therefore, GPIHBP1 could be a functional gene for milk fat content, and in particular, the G to A mutation at chr14:2553998 in the promoter region of GPIHBP1 could be a functional mutation for its effects on milk fat content.

\section{Methods}

Animals. Seventeen Chinese Holstein cows in the same period of lactation were selected from the Beijing Sanyuan Dairy Farm Center. All of the cows were fed under consistent environmental conditions. Mammary gland tissues were collected from each cow within $30 \mathrm{~min}$ after slaughter and stored in liquid nitrogen. The entire procedure for the collection of the tissue samples from all animals was performed in strict accordance with the protocol approved by the Animal Welfare Committee of China Agricultural University (Permit number: DK996).

Culture of BMECs and 293T cells. BMECs were plated in serum-containing medium DMEM-F12 supplemented with 10\% fetal bovine serum (FBS) (Invitrogen, USA), 1\% Insulin-Transferrin-Selenium-Sodium Pyruvate (ITS-A) (Invitrogen, USA), $10 \mathrm{ng} / \mathrm{ml}$ of epidermal growth factor and $100 \mathrm{units} / \mathrm{ml}$ penicillin and streptomycin. The digested cells were cultured at $37^{\circ} \mathrm{C}$ in a humidified atmosphere containing $5 \% \mathrm{CO}_{2}$.

$293 \mathrm{~T}$ cells were plated in the following serum-containing medium: DMEM supplemented with 5\% FBS (Invitrogen, USA) and 100 units $/ \mathrm{ml}$ penicillin and streptomycin. The digested cells were cultured at $37^{\circ} \mathrm{C}$ in a humidified atmosphere containing $5 \% \mathrm{CO}_{2}$.

RNA extraction and reverse transcription. Total RNA was extracted from mammary gland tissues or cells using Trizol (Invitrogen, USA), and each sample was reverse transcribed to cDNA in a $40 \mu \mathrm{L}$ reaction using the Prime Script RT reagent Kit (Takara Biotechnology, Tokyo, Japan).

Construction of the recombinant pCDNA 3.1(+)-GPIHBP1 eukaryotic expression vector. According to mRNA sequence data for the bovine GPIHBP1 gene, a pair of specific primers containing Nhe I and EcoR I restriction enzyme cutting sites was designed for amplification of the ORF of the bovine GPIHBP1 gene using Oligo 6.0 software (forward 5'-CTTTgctagcCCCCGTAGGATGAAGGCA- $3^{\prime}$ and reverse $5^{\prime}$-AGGgaattcTCAGAGCCCCATCTCCTG- $3^{\prime}$ ). PCR was performed under the following conditions: after denaturation at $95^{\circ} \mathrm{C}$ for $5 \mathrm{~min}$, DNA amplification was performed for 35 cycles at $95^{\circ} \mathrm{C}$ for $30 \mathrm{~s}, 61^{\circ} \mathrm{C}$ for $30 \mathrm{~s}$, and $72^{\circ} \mathrm{C}$ for $1 \mathrm{~min}$, with a final extension at $72^{\circ} \mathrm{C}$ for $7 \mathrm{~min}$. The obtained PCR product was purified using the Omega E.Z.N.A. cycle Pure Kit (Omega, USA).

Plasmid pcDNA 3.1(+) was purchased from Life Technologies (USA) and was transformed into E.coli DH5 $\alpha$ competent cells (Tiangen Biotech, China) for amplification. Then, the pcDNA3.1 $(+)$ vector was isolated from transformants using the AxyPrep Plasmid Miniprep Kit (Axygen, USA). Next, the pcDNA 3.1(+) eukaryotic expression vector and the purified PCR production were digested with the Nhe I and EcoR I restriction enzyme at $37^{\circ} \mathrm{C}$ for $4 \mathrm{~h}$. After purification of the digestion products using the Omega E.Z.N.A. Cycle Pure Kit, bovine GPIHBP1 cDNA was ligated into the pcDNA3.1(+) eukaryotic expression vector using T4 DNA ligase (NEB, USA). Recombinant pcDNA 3.1(+)-GPIHBP1 was amplified in E. coli DH5 $\alpha$ competent cells (Tiangen Biotech, China) and isolated with the AxyPrep Plasmid Miniprep Kit (Axygen, USA). The correct sequence of the pcDNA3.1(+)-GPIHBP1 plasmid was verified via restriction enzyme mapping and DNA sequencing.

Transfection of pcDNA 3.1(+)-GPIHBP1 into BMECs. After transformation into $E$. coli DH5 $\alpha$ competent cells, pcDNA 3.1(+)-GPIHBP1 and pcDNA 3.1(+) were isolated with the E.Z.N.A. Endo-Free Plasmid Mini Kit I (Omega, USA). The cells were passaged and plated in 6-well plates for 24 hours before transfection at $80 \%$ to $90 \%$ confluence. The cells were divided into two groups: a transfection reagent + pcDNA3.1 $(+)($ Control) group and a transfection reagent + pcDNA 3.1(+)-GPIHBP1 group (Over expression). The recombinant pcDNA 3.1(+)-GPIHBP1 eukaryotic expression vector or the pcDNA3.1 $(+)$ plasmid was transiently transfected into bovine primary mammary epithelial cells according to the instructions for the Roche X-treme GENE HP DNA transfection reagent. Transfection was performed using a 12:1 ratio of the Roche X-treme GENE HP DNA transfection reagent (Roche, USA) $(\mu \mathrm{l})$ to the vector $(\mu \mathrm{g})$. At 36 hours after transfection, the cells were collected to detect GPIHBP1 mRNA expression levels via real-time quantitative PCR.

Silencing of GPIHBP1 via RNAi in BMECs. Stealth ${ }^{\mathrm{TM}}$ RNAi siRNA (GACGGAUCUCUGACGACCAUAUCCU) targeting the bovine GPIHBP1 gene open reading frame was designed using BLOCK-iT ${ }^{\mathrm{TM}}$ RNAi Designer (Life Technologies, USA) and synthesized by Life Technologies (USA). The Stealth ${ }^{\text {TM }}$ RNAi siRNA negative control (Med GC) was purchased from Life Technologies (USA) and used as a control for sequence-independent effects.

One day prior to transfection, cells were seeded without antibiotics and exhibited a density of $80 \%$ at the time of transfection. GPIHBP1-siRNA or the Stealth ${ }^{\mathrm{TM}}$ RNAi siRNA Negative Control was transfected into bovine primary mammary epithelial cells using the Roche X-treme GENE siRNA Transfection Reagent (Roche, USA) according to the manufacturer's instructions. Transfection was performed using a 10:1 ratio of the X-treme GENE 
siRNA Transfection Reagent $(\mu \mathrm{l})$ to siRNA $(\mu \mathrm{g})$. Cells were harvested at 48 hours after transfection for mRNA analysis via real-time quantitative PCR.

Real-time quantitative PCR (qPCR). The expression levels of GPIHBP1 and several other milk fat-related genes ( $L P L, C D 36, V L D L R, A C A C A$, and FASN) were detected using real-time quantitative PCR. qPCR primers were designed using the Primer 3 web-tool (http://frodo.wi.mit.edu/primer3/) and Oligo 6.0 software and are shown in Table S2. All qPCR procedures were performed using LightCycler $480^{\circledR}$ SYBR Green I Master on a Roche LightCycler $480^{\circledR}$ instrument. Real-time PCR amplification was performed in a 96-well plate in a total volume of $20 \mu \mathrm{L}$ containing the following reagents: $1 \mu \mathrm{L}$ of cDNA, $1 \mu \mathrm{L}(10 \mathrm{pM} / \mu \mathrm{L})$ of both the forward primer and reverse primer, $10 \mu \mathrm{L}$ of Blue-SYBR-Green Mix $(2 \times)$ and water (Roche Applied Science, USA). All qPCRs for each sample were performed in triplicate, and the relative mRNA expression levels were normalized to the housekeeping gene glyceraldehyde phosphate dehydrogenase $(G A P D H)$ via the $2^{-\triangle \triangle C T}$ method $^{24}$.

SNP detection. Seven pairs of primers (see Supplementary Table S3) were designed to investigate the SNPs of GPIHBP1. These primers covered all exons, introns, and 3-kb upstream sequences (as promoter region) of GPIHBP1. DNA was extracted from semen samples from 13 bulls. PCR was then performed for each sample, and the obtained PCR products were sequenced using ABI3730XL. All SNPs were detected based on comparison with the reference genome using the DNAMAN software (Lynnon BioSoft, QC, Canada).

Measurement of GPIHBP1 promoter activity. Amplification of GPIHBP1 promoter regions. We detected four SNPs in the promoter region of the bovine GPIHBP1 gene, which were completely linked with only two haplotypes in the test population. Based on this finding, PCR primers (Table S4) containing Kpn I and Bg1 II restriction enzyme cutting sites were designed to amplify four different bovine GPIHBP1 gene promoter fragments, designated P1 ( -509 to -49$)$, P2 ( -1440 to -49$)$, P3 ( -1673 to -49$)$, and P4 ( -2204 to -49$)$, which contained one, two, three, and four SNP sites, respectively. PCR was performed under the following conditions: after denaturation at $95^{\circ} \mathrm{C}$ for $5 \mathrm{~min}$, DNA amplification was performed for 38 cycles at $95^{\circ} \mathrm{C}$ for $30 \mathrm{~s}, 70^{\circ} \mathrm{C}$ for $1 \mathrm{~min}$, and $72^{\circ} \mathrm{C}$ for $2 \mathrm{~min}$, with a final extension at $72^{\circ} \mathrm{C}$ for $7 \mathrm{~min}$. The PCR product was purified with the Omega E.Z.N.A. cycle Pure Kit (Omega, USA). Next, the PGL4.14 vector was transformed into E. coli DH5 $\alpha$ competent cells for amplification and then isolated using an AxyPrep Plasmid Miniprep Kit (Axygen, USA).

Construction of recombinant promoter vectors. PGL4.14 and the purified PCR products were digested with the $\mathrm{Kpn} \mathrm{I}$ and $\mathrm{Bg} 1 \mathrm{II}$ restriction enzyme at $37^{\circ} \mathrm{C}$ for $4 \mathrm{~h}$. After purification of the digested products using the Omega E.Z.N.A. Cycle Pure Kit, the GPIHBP1 promoter fragments were ligated into the PGL4.14 vector using T4 DNA ligase (NEB, USA). The recombinant vectors were amplified in E. coli DH5 $\alpha$ competent cells and isolated with the AxyPrep Plasmid Miniprep Kit. Finally, restriction enzyme mapping and DNA sequencing were performed to determine the correct sequences of the eight types of recombinant promoters (P1-Wild, P1-Mutation, P2-Wild, P2-Mutation, P3-Wild, P3-Mutation, P4-Wild, and P4-Mutation).

Luciferase reporter assay. After transformation into E. coli DH5 $\alpha$ competent cells, PGL4.14, pRL-TK and the eight types of recombinant promoter vectors were isolated with the E.Z.N.A. Endo-Free Plasmid Mini Kit I (Omega, USA). Then, 293 T cells were cultured in 24 -well tissue culture plates overnight, and transfections were performed using Lipofectamine ${ }^{\mathrm{TM}} 2000$ (Life Technologies, USA). The cells were divided into nine groups: PGL4.14 + pRL-TK + Lip2000 (Negative control), P1-Wild + pRL-TK + Lip2000 (P1-W), P1-Mutation + pRL-TK + Lip2000 (P1-M), P2-Wild + pRL-TK + Lip2000 (P2-W), P2-Mutation + pRL-TK + Lip2000 (P2-M), P3-Wild + pRL-TK + Lip2000 (P3-W), P3-Mutation + pRL-TK + Lip2000 (P3-M), P4-Wild + pRL-TK + Lip2000 (P4-W), and $\mathrm{P} 4-$ Mutation + pRL-TK + Lip2000 (P4-M). According to the manufacturer's instructions of the Luciferase Reporter Assay system, transfections were performed using a 9:1 ratio of PGL4.14 or the recombinant promoter vector $(\mu \mathrm{g})$ to the $\mathrm{pRL}-\mathrm{TK}$ vector $(\mu \mathrm{g})$ and repeated at least three times for each construct. At 24 hours after transfection, the cells were harvested, and firefly and Renilla luciferase activities were analyzed. To perform normalization for the transfection efficiency, the firefly luciferase value was divided by the Renilla luciferase value for the same sample. All assays were repeated at least three times.

In vitro experiments. The ACGA and GAAG promoter vectors were generated from P4-W(GCGA) and $\mathrm{P} 4-\mathrm{M}(\mathrm{AAAG})$ by creating one point mutations using QuikChange ${ }^{\circledR}$ Site-Directed Mutagenesis Kit (Stratagene, CA, USA) according to the manufacturer's instructions. Two pairs of primers were designed for site-directed mutagenesis (see Supplementary Table S5). All changes were confirmed by sequencing. The promoter activities of plasmids with four different haplotypes (GCGA, ACGA, AAAG and GAAG) were measured using a dual-luciferase reporter system and the experimental procedure was the same as described in luciferase reporter assay.

EMSA (electrophoretic mobility shift assay). The mammary gland tissues of three cows were used to isolate nuclear protein. Oligonucleotide probes were labeled with biotin at the $5^{\prime}$ ends. For competition assays, non-biotin-labeled oligonucleotides were used as cold probes and were added to the binding reaction. The sequences of the double-stranded oligonucleotides used for electrophoretic mobility shift assays (EMSAs) are listed in Table S6. The EMSAs were performed using Viagene's non-radioactive EMSA kits.

Association analysis. For the association analysis between the identified GPIHBP1 SNP or the K232A mutation in DGAT1 and milk fat percentage, 6619 cows were used. These cows were different from the cows 
that we previously used for GWAS ${ }^{12}$ and came from 126 sire families. Sequenom MassARRAY iPLEX genotyping technology was employed for the genotyping of both SNPs. The estimated breeding value (EBV) of the cows for the milk fat percentage, which was provided by the Chinese Dairy Association, was then converted to de-regressed proofs (DRP) using the method proposed by Garrick et al. ${ }^{25}$. The association analysis was first performed for the two SNPs separately based on the following model: $\mathrm{DRP}=\mu+$ sire family (random) + genotype (GPIHBP1 or DGAT1) + error. Then, a joint analysis was performed for both SNPs based on the following model: $\mathrm{DRP}=\mu+$ sire family $($ random $)+$ genotype $(G P I H B P 1)+$ genotype $(D G A T 1)+$ interaction $(G P I H B P 1 \times$ $D G A T 1)+$ error. The SAS procedure GLM was employed for the analysis.

Haplotype analysis. We genotyped the SNPs detected in GPIHBP1 in 158 randomly selected individuals via PCR and performed haplotype analysis for these SNPs. In addition, we performed haplotype analysis for the SNP in GPIHBP1 and the SNP K232A in DGAT1 in the 6619 cows. The software PHASE v2.1.1 ${ }^{14,15}$ was used for the analysis.

\section{References}

1. Beigneux, A. P., Davies, B. S., Bensadoun, A., Fong, L. G. \& Young, S. G. GPIHBP1, a GPI-anchored protein required for the lipolytic processing of triglyceride-rich lipoproteins. J Lipid Res 50(Suppl), S57-62, doi:10.1194/jlr.R800030-JLR200 (2009).

2. Young, S. G. et al. GPIHBP1: an endothelial cell molecule important for the lipolytic processing of chylomicrons. Curr Opin Lipidol 18, 389-396, doi:10.1097/MOL.0b013e3281527914 (2007).

3. Gin, P. et al. The acidic domain of GPIHBP1 is important for the binding of lipoprotein lipase and chylomicrons. J Biol Chem $\mathbf{2 8 3}$, 29554-29562, doi:10.1074/jbc.M802579200 (2008).

4. Dallinga-Thie, G. M. et al. The metabolism of triglyceride-rich lipoproteins revisited: new players, new insight. Atherosclerosis 211, 1-8, doi:10.1016/j.atherosclerosis.2009.12.027 (2010).

5. Beigneux, A. P. et al. Assessing the role of the glycosylphosphatidylinositol-anchored high density lipoprotein-binding protein 1 (GPIHBP1) three-finger domain in binding lipoprotein lipase. J Biol Chem 286, 19735-19743, doi:10.1074/jbc.M111.242024 (2011).

6. Davies, B. S. et al. Assessing mechanisms of GPIHBP1 and lipoprotein lipase movement across endothelial cells. J Lipid Res 53, 2690-2697, doi:10.1194/jlr.M031559 (2012).

7. Young, S. G. \& Zechner, R. Biochemistry and pathophysiology of intravascular and intracellular lipolysis. Genes Dev 27, 459-484, doi:10.1101/gad.209296.112(2013).

8. Weinstein, M. M. et al. Abnormal patterns of lipoprotein lipase release into the plasma in GPIHBP1-deficient mice. J Biol Chem 283, 34511-34518, doi:10.1074/jbc.M806067200 (2008).

9. Olivecrona, G. et al. Mutation of conserved cysteines in the Ly6 domain of GPIHBP1 in familial chylomicronemia. J Lipid Res 51, 1535-1545, doi:10.1194/jlr.M002717 (2010)

10. Hernell, O. \& Olivecrona, T. Human milk lipases. I. Serum-stimulated lipase. J Lipid Res 15, 367-374 (1974).

11. Beigneux, A. P. et al. GPIHBP1 and lipolysis: an update. Curr Opin Lipidol 20, 211-216, doi:10.1097/MOL.0b013e32832ac026 (2009).

12. Jiang, L. et al. Genome wide association studies for milk production traits in Chinese Holstein population. PLoS One 5, e13661, doi:10.1371/journal.pone.0013661 (2010).

13. Jiang, L. et al. Targeted resequencing of GWAS loci reveals novel genetic variants for milk production traits. BMC Genomics 15, 1105, doi:10.1186/1471-2164-15-1105 (2014).

14. Stephens, M., Smith, N. J. \& Donnelly, P. A new statistical method for haplotype reconstruction from population data. Am J Hum Genet 68, 978-989, doi:10.1086/319501 (2001).

15. Stephens, M. \& Donnelly, P. A comparison of bayesian methods for haplotype reconstruction from population genotype data. Am J Hum Genet 73, 1162-1169, doi:10.1086/379378 (2003).

16. Cases, S. et al. Identification of a gene encoding an acyl CoA:diacylglycerol acyltransferase, a key enzyme in triacylglycerol synthesis. Proceedings of the National Academy of Sciences of the United States of America 95, 13018-13023 (1998).

17. Williams, K. J. Some Things Just Have to Be Done In Vivo GPIHBP1, Caloric Delivery, and the Generation of Remnant Lipoproteins. Arterioscler Thromb Vasc Biol 29, 792-795 (2009).

18. Beigneux, A. P. et al. Chylomicronemia with a mutant GPIHBP1 (Q115P) that cannot bind lipoprotein lipase. Arterioscler Thromb Vasc Biol 29, 956-962, doi:10.1161/ATVBAHA.109.186577 (2009).

19. Charriere, S. et al. GPIHBP1 C89F neomutation and hydrophobic C-terminal domain G175R mutation in two pedigrees with severe hyperchylomicronemia. J Clin Endocrinol Metab 96, E1675-1679, doi:10.1210/jc.2011-1444 (2011).

20. Beigneux, A. P. et al. Glycosylphosphatidylinositol-anchored high-density lipoprotein-binding protein 1 plays a critical role in the lipolytic processing of chylomicrons. Cell Metab 5, 279-291, doi:10.1016/j.cmet.2007.02.002 (2007).

21. Matsumoto, H. et al. The SNPs in the ACACA gene are effective on fatty acid composition in Holstein milk. Mol Biol Rep 39, 8637-8644, doi:10.1007/s11033-012-1718-5 (2012).

22. Badaoui, B. et al. Goat acetyl-coenzyme A carboxylase alpha: molecular characterization, polymorphism, and association with milk traits. J Dairy Sci 90, 1039-1043, doi:10.3168/jds.S0022-0302(07)71590-4 (2007).

23. Roy, R. et al. Association of polymorphisms in the bovine FASN gene with milk-fat content. Anim Genet 37, 215-218, doi:10.1111/ j.1365-2052.2006.01434.x (2006).

24. Livak, K. J. \& Schmittgen, T. D. Analysis of relative gene expression data using real-time quantitative PCR and the 2(-Delta Delta C(T)) Method. Methods 25, 402-408, doi:10.1006/meth.2001.1262 (2001).

25. Garrick, D. J., Taylor, J. F. \& Fernando, R. L. Deregressing estimated breeding values and weighting information for genomic regression analyses. Genet Sel Evol 41, 55, doi:10.1186/1297-9686-41-55 (2009).

\section{Acknowledgements}

The work was supported by a grant from the National Natural Science Foundation of China (31201772), the Program of New Breed Development via Transgenic Technology (2014ZX0800953B), Chinese Universities Scientific Fund (2014XJ003) and the Program for Changjiang Scholars and Innovative Research Team in University (IRT_15R62). The funders had no role in the study design, data collection and analysis, decision to publish or preparation of the manuscript. 


\section{Author Contributions}

L.J. conceived and designed the experiments. J.Y., X.L. and H.F.W. performed the experiments. D.W., C.N. and Q.Z. analyzed the data. J.Y. wrote the paper. Q.Z. and L.J. provided editorial suggestions and revisions. All authors read and approved the final manuscript.

\section{Additional Information}

Supplementary information accompanies this paper at doi:10.1038/s41598-017-08668-6

Competing Interests: The authors declare that they have no competing interests.

Publisher's note: Springer Nature remains neutral with regard to jurisdictional claims in published maps and institutional affiliations.

(c) (i) Open Access This article is licensed under a Creative Commons Attribution 4.0 International License, which permits use, sharing, adaptation, distribution and reproduction in any medium or format, as long as you give appropriate credit to the original author(s) and the source, provide a link to the Creative Commons license, and indicate if changes were made. The images or other third party material in this article are included in the article's Creative Commons license, unless indicated otherwise in a credit line to the material. If material is not included in the article's Creative Commons license and your intended use is not permitted by statutory regulation or exceeds the permitted use, you will need to obtain permission directly from the copyright holder. To view a copy of this license, visit http://creativecommons.org/licenses/by/4.0/.

(C) The Author(s) 2017 